\title{
The Dynamics of Mixed Layer Deepening during Open-Ocean Convection
}

\author{
TAIMOOR SOHAIL \\ Research School of Earth Sciences, and ARC Centre of Excellence for Climate Extremes, Australian National University, \\ Canberra, Australian Capital Territory, Australia \\ BISHAKHDATTA GAYEN \\ Department of Mechanical Engineering, University of Melbourne, Melbourne, Australia and Centre for Atmospheric and \\ Oceanic Sciences, Indian Institute of Science, Bangalore, India \\ ANDREW MCC. HOGG \\ Research School of Earth Sciences, and ARC Centre of Excellence for Climate Extremes, Australian National University, \\ Canberra, Australian Capital Territory, Australia
}

(Manuscript received 31 October 2019, in final form 25 March 2020)

\begin{abstract}
Open-ocean convection is a common phenomenon that regulates mixed layer depth and ocean ventilation in the high-latitude oceans. However, many climate model simulations overestimate mixed layer depth during open-ocean convection, resulting in excessive formation of dense water in some regions. The physical processes controlling transient mixed layer depth during open-ocean convection are examined using two different numerical models: a high-resolution, turbulence-resolving nonhydrostatic model and a large-scale hydrostatic ocean model. An isolated destabilizing buoyancy flux is imposed at the surface of both models and a quasi-equilibrium flow is allowed to develop. Mixed layer depth in the turbulence-resolving and largescale models closely aligns with existing scaling theories. However, the large-scale model has an anomalously deep mixed layer prior to quasi-equilibrium. This transient mixed layer depth bias is a consequence of the lack of resolved turbulent convection in the model, which delays the onset of baroclinic instability. These findings suggest that in order to reduce mixed layer biases in ocean simulations, parameterizations of the connection between baroclinic instability and convection need to be addressed.
\end{abstract}

\section{Introduction}

Open-ocean convection is a climatically important process that commonly occurs in high-latitude regions of the ocean. Localized open-ocean convection events are triggered by a destabilizing surface buoyancy flux that comes about through changes in the general circulation (Martinson et al. 1981) and atmospheric conditions (Clarke and Gascard 1983; Cheon et al. 2014). The destabilizing surface buoyancy flux creates an unstable vertical buoyancy gradient (dense fluid overlying lighter fluid) that in turn incites the development of rapid vertical convective plumes that communicate the surface forcing signal through depth. Vertical mixing during open-ocean convection leads to the homogenization of surface layer properties, creating a deep mixed layer

Corresponding author: Taimoor Sohail, t.sohail@unsw.edu.au
(Marshall and Schott 1999). The mixed layer (also referred to here as a mixed patch) plays a role in modulating the properties of dense overflows in the Southern Ocean and therefore the downwelling branch of the global meridional overturning circulation (MOC), including the Atlantic meridional overturning circulation (AMOC) (Talley 1999; Danabasoglu et al. 2012). The AMOC has widespread consequences on the global climate, such as modulating global ocean heat content (Kostov et al. 2014), global marine biological activity (Schmittner 2005) and carbon uptake (Sallée et al. 2012). Therefore, open-ocean convection (and the deep mixed layer that forms as a consequence of the process) plays a major role in regulating the global climate.

Given the importance of open-ocean convection in the global climate system, understanding the dynamics of the process and representing it faithfully in ocean models is critical. A number of observational, 
numerical, and experimental studies have been conducted to investigate the processes at work during an open-ocean convection event. Field studies have identified hot spots of open-ocean convection in the Labrador (Clarke and Gascard 1983), Mediterranean (Schott and Leaman 1991), and Weddell Seas (Zwally and Gloersen 1977; Jena et al. 2019). In addition, numerous idealized numerical studies have been conducted of rotating open-ocean convection with the presence of a background linear stratification (Sander et al. 1995; Visbeck et al. 1996; Legg et al. 1996; Jones and Marshall 1997; Cui and Street 2001). Large-scale ocean and coupled climate modeling studies have also recreated deep ocean convection events which share qualitatively similar flow patterns. For instance, Cheon et al. $(2014,2015)$ and Kurtakoti et al. (2018) investigated the driving mechanisms of the Weddell Sea Polynya using large-scale ocean and climate models and found that atmospheric conditions, the general circulation and bottom topography all play leadingorder roles in the development of open-ocean convection events. Coupled with laboratory experiments by Ivey et al. (1995), Coates et al. (1995), and Jacobs and Ivey (1999), among others, these numerical studies have identified three phases of open-ocean convection: preconditioning, deep convection, and lateral exchange and spreading (see Marshall and Schott 1999 for more details).

In the preconditioning phase, changes in circulation patterns and air-sea interactions encourage the development of a weak destabilizing surface buoyancy flux and a doming of surface isopycnals. Winds, temperature, and sea ice formation all play a role (to varying degrees, depending on the region) in generating this buoyancy flux, which weakens the vertical stratification in the surface boundary layer. Over time, the surface buoyancy flux strengthens, triggering deep convection, the threedimensional, small-scale, and rapid exchange of water properties between the surface and deep ocean through vertical mixing. The dominant form of heat transport during the deep convection phase is vertical buoyancy transport due to turbulent convective plumes. Deep convection leads to the formation of a homogeneous mixed patch beneath the unstable buoyancy forced region extending up to $2000 \mathrm{~m}$ below the ocean surface (Marshall and Schott 1999). Deep convection also enhances the lateral gradient between the mixed patch and the surrounding ambient, initiating an azimuthal geostrophic current. Arising from the thermal wind balance, this current advects fluid around the edge of the mixed patch (Cui and Street 2001). Provided the radius of the cooling patch is larger than the local Rossby deformation radius, the geostrophic current is unstable to baroclinic instability and breaks down, giving way to baroclinic eddies. The breakup of the geostrophic current and formation of baroclinic eddies occurs during the lateral exchange and spreading phase. Baroclinic eddies draw the well-mixed fluid out toward the ambient, stratified region. The effect of baroclinic eddies is modulated somewhat by the strength of the vertical convection in the system (Callies and Ferrari 2018). That said, in the parameter regime relevant to high-latitude open-ocean convection, the development of baroclinic eddies halts the convective deepening of the mixed layer. Upon the cessation of conditions favorable to deep convection, baroclinic eddies rapidly destroy the mixed patch (Jones and Marshall 1997; Katsman et al. 2004; Gelderloos et al. 2011). Baroclinic eddies dominate the buoyancy transport during the lateral exchange phase, such that lateral buoyancy fluxes overwhelm the vertical buoyancy flux due to convection (Marshall and Schott 1999).

The three phases of open-ocean convection illustrate that the process is driven by flow features that cover a large range of temporal and spatial scales. As a consequence, accurately characterizing open-ocean convection and subsequent mixed layer deepening in large-scale ocean models is challenging. Smaller-scale flow processes, particularly convection and baroclinic eddies, are often parameterized in models. Deep convection is represented by one-dimensional parameterizations, including through the explicit enhancement of vertical diffusivity (Klinger et al. 1996) and penetrative mixing schemes (Large et al. 1994). Mixed layer eddies and restratification of the surface boundary layer are represented by lateral and vertical mixing schemes, including $K$-profile parameterization (KPP) (Large et al. 1994), energetic planetary boundary layer (ePBL) (Reichl and Hallberg 2018), and the mixed layer eddy parameterization proposed by FoxKemper et al. (2008).

However, present parameterizations of convection may lead to the development of an anomalously deep mixed layer following deep convection in some global ocean models (Ferrari and Paparella 2003; Ilicak et al. 2014; Courtois et al. 2017). For instance, recent reviews of CORE-II simulations have found that winter mixed layer depths are consistently overestimated in regions of open-ocean convection, such as the Labrador and Nordic Seas and parts of the Southern Ocean, regardless of model type and parameterization employed (Danabasoglu et al. 2014; Farneti et al. 2015). The deep mixed layer bias is particularly concerning in the Southern Ocean, where small variations in mixed layer deepening can entrain warm, salty Circumpolar Deep Water (CDW) into cold, fresh surface waters. 
Entrainment of the CDW into the cold surface waters prevents the formation of sea ice, resulting in stronger surface buoyancy fluxes and a persistent polynya characterized by rapid convective deepening and dense water formation. The mixed layer depth bias can therefore have a significant impact on the downwelling branch of the MOC. As a consequence, it is important to identify the cause of the mixed layer depth bias during deep convection in ocean models.

Studies looking to address the mixed layer depth bias in high-latitude regions have focused in part on pursuing improved scaling theories of mixed layer growth. Turner (1973) manipulated the one-dimensional buoyancy budget for convective deepening to obtain a scaling for transient mixed layer growth during deep convection under the assumption that convection is nonpenetrative. Subsequent laboratory experiments have confirmed that the nonpenetrative assumption is first-order accurate for penetrative convection in open-ocean convection events (Heidt 1977; Marshall and Schott 1999; Levy and Fernando 2002). Visbeck et al. (1996) further used the dominant buoyancy balance during the lateral exchange and spreading phase to develop scaling predictions for quasi-equilibrium mixed layer depth and the time at which quasi-equilibrium is reached. The scaling prediction developed by Visbeck et al. (1996) has been validated by a number of laboratory (see Visbeck et al. 1996 for details) and numerical studies (Jones and Marshall 1997; Fox-Kemper et al. 2008). While these scaling theories have been widely incorporated into large-scale ocean models, to the best of the authors' knowledge, they have not been rigorously tested against a nonhydrostatic, turbulence-resolving, three-dimensional numerical simulation of open-ocean convection.

The dominant physical processes during deep convection have also been scrutinized as a means to correct the anomalously deep winter mixed layers in ocean models. In particular, idealized research has highlighted the importance of representing the spatial and temporal variability of turbulent convection in ocean models. Sohail et al. (2019) concluded that temporally variant flow in the Southern Ocean (including convection, turbulence and submesoscale eddies) dominates dense water formation in the lower overturning cell of the MOC. Ilicak et al. (2014) found that models that do not accurately resolve the spatial heterogeneity of convective plumes exhibit anomalously deep mixed layers at high latitudes. A high-resolution climate modeling study by Kurtakoti et al. (2018) also found that adequately resolving bottom topographic features in the Weddell Sea is important in recreating the intermittent Weddell Sea Polynya formation observed in the historical record.
In addition, Kuhlbrodt and Monahan (2003) found that introducing a stochastic variability to buoyancy forcing during deep convection can significantly alter the duration of an open-ocean convection event, and by extension, dense water formation.

In this work, we compare the representation of convection in a nonhydrostatic turbulence-resolving simulation with convection in a hydrostatic, largescale ocean model. The turbulence-resolving simulation resolves all scales of motion, from the turbulent Kolmogorov and Batchelor scales to the basin scale, allowing us to identify the role of specific processes during open-ocean convection. The large-scale simulation has a model configuration analogous to the turbulence-resolving simulations and is run with two mixing schemes (KPP and ePBL). Using the turbulenceresolving simulations, we validate existing open-ocean convection scaling theories and identify possible causes of mixed layer depth biases in large-scale ocean models. In section 2, we provide an overview of the models used and the suite of simulations conducted. In section 3, we summarize derivations for existing theoretical scalings. In section 4 , we identify the dominant flow features during open-ocean convection, test the validity of existing scaling theories, and examine the role of turbulence during deep convection. In section 5, we lay out a roadmap to improving the representation of mixed layer deepening during open-ocean convection based on our findings and summarize our key results and conclusions.

\section{Method}

\section{a. Model setup}

In this study, we set up an idealized open-ocean convection event in a reentrant channel (Fig. 1). The channel has dimensions $H \times L \times W$, corresponding to the $z$ (depth), $x$ (zonal), and $y$ (meridional) directions, respectively. The Coriolis parameter $f$ varies latitudinally as $f(y)=f_{0}+\beta y$, where $f_{0}=f(y=0)$ and $\beta=d f / d y$. The surface destabilizing buoyancy forcing is represented by a Gaussian spot (see blue-gray contour in Fig. 1) with heat flux, $\mathbb{H}=B_{0} \exp \left\{-0.5\left[(x-l)^{2} / n^{2}+\right.\right.$ $\left.\left.(y-m)^{2} / n^{2}\right]\right\}$, where $B_{0}$ is the maximum heat flux, $(l, m)$ are the coordinates of the center of the Gaussian spot, and $n$ is a constant controlling the radius $R$ of the destabilizing region. Note that this pattern of imposed buoyancy differs from most numerical and laboratory studies of open-ocean convection, which utilize a uniform disk-shaped cooling signal (e.g., Maxworthy and Narimousa 1994; Coates et al. 1995; Noh et al. 2003). A Gaussian buoyancy profile is a more realistic representation of the surface flux forcing of the ocean. This profile leads to the "doming" of isopycnals that 


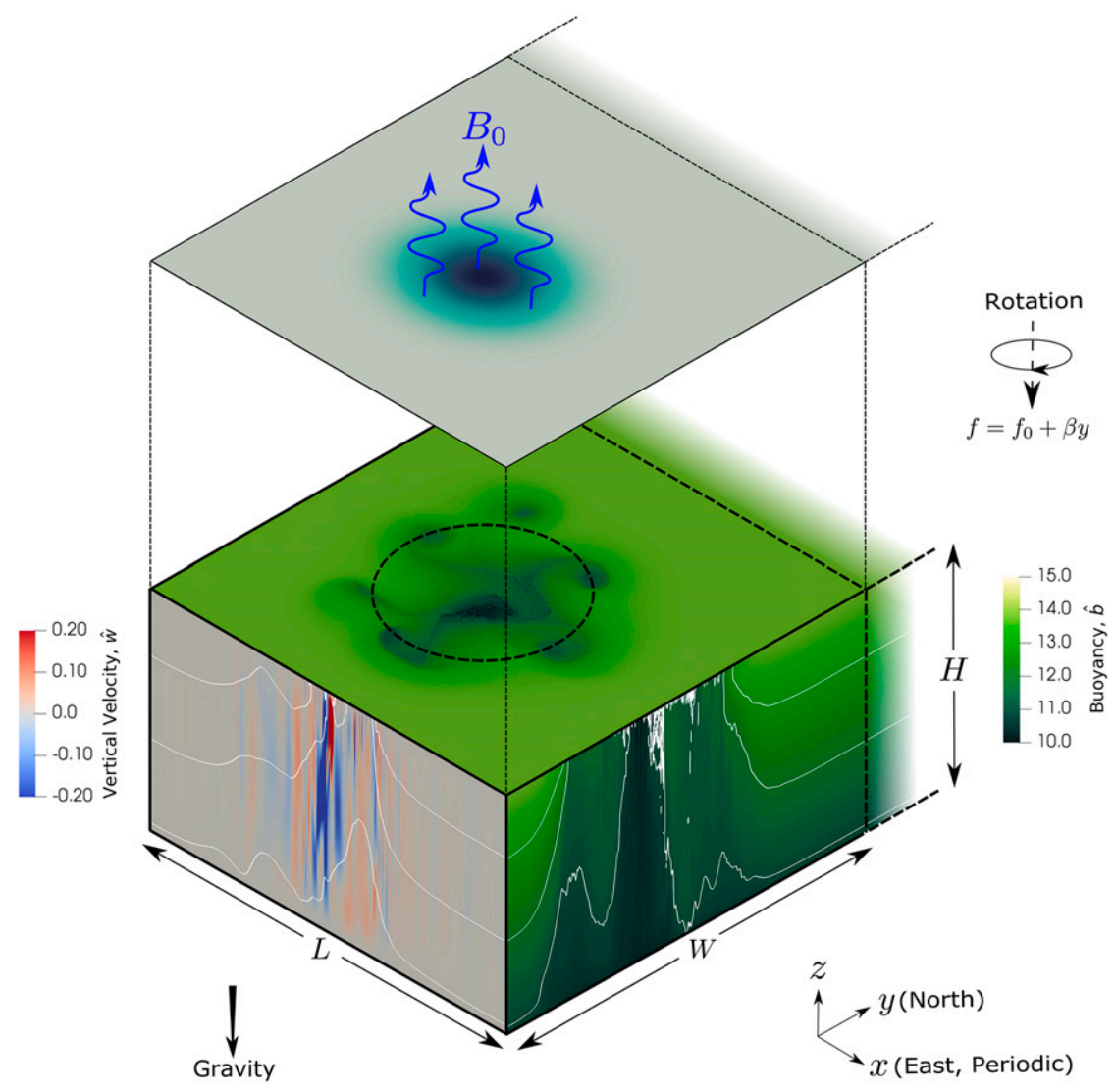

FIG. 1. An overview of the model domain, with overlaid flow solutions from the DNS for $\mathrm{Ra}_{f}=9 \times 10^{8}, \hat{N}=4.10$, and $\hat{t}=355.9$. The upper $x-y$ plane (in blue-gray) depicts the Gaussian surface heat flux $\mathbb{H}$. The lower $x-y$ plane (in green-yellow) shows the normalized buoyancy $\hat{b}$ at $z / H=0.98$. The dashed black circle indicates the location of the destabilizing buoyancy source at the model surface. The $y-z$ plane shows the buoyancy $\hat{b}$ at $x / L=0.5$. The $z-x$ plane depicts the normalized vertical velocity $\hat{w}$ at $y / W=0.5$. White contours correspond to isopycnals of value $\hat{b}=[13,12,11]$ from top to bottom, respectively. The dashed and dotted lines at $y / W \geq 1$ indicate the presence of a sponge layer. Buoyancy is defined as $b=g \alpha T$, where $T$ is the temperature field.

occurs during the preconditioning phase and may be driven either by a vortex-like wind field or the surface flux forcing during the convective event (Straneo and Kawase 1999). All simulations are spun up with a linear ambient stratification $N$, defined as $N=\sqrt{g \alpha \partial T / \partial z}$, where $g$ is the acceleration due to gravity, $\alpha$ is the (constant) thermal expansion coefficient, and $\partial T / \partial z$ is the background vertical thermal gradient (in the linearly stratified system discussed here, $\partial T / \partial z=\Delta T / H$, where $\Delta T$ is the vertical temperature difference). Assuming a linear equation of state, the maximum heat flux $B_{0}$ is calculated as $B_{0}=g \alpha Q / \rho_{0} c_{p}=g \alpha \kappa \partial T / \partial z$, where $\rho_{0}$ is the reference density, $c_{p}$ is the specific heat of the fluid, $\kappa$ is the background diffusivity, and $Q$ is the heat flux per unit area $\left(\mathrm{W} \mathrm{m}^{-2}\right)$. The idealized setup is used in two models: a turbulence-resolving direct numerical simulation (DNS), and the GFDL Modular Ocean
Model version 6 (MOM6; see Adcroft et al. 2019 for model details).

\section{1) DiRECT NUMERICAL SIMULATIONS}

The DNS solves the incompressible, nonhydrostatic Navier-Stokes momentum equations with a Boussinesq approximation, linear equation of state, beta-plane assumption, and conservation of heat and mass in a rotating reference frame (see Sohail et al. 2018). The simulations are conducted with a grid of size $\left[N_{x}, N_{y}\right.$, $\left.N_{z}\right]=[1024,513,257]$ grid points in the $x, y$, and $z$ directions, respectively. The grid is clustered in the vertical $(z)$ direction to resolve the surface diffusive boundary layer and bottom Ekman layer, and in the meridional $(y)$ direction to resolve the convecting patch. The grid is uniform in the zonally reentrant periodic $(x)$ direction. Typically, the grid resolution in 
DNS is such that all scales of motion in the flow are resolved, from the turbulent Kolmogorov scale, $L_{k}=$ $\left(\nu^{2} / \varepsilon^{\prime}\right)^{1 / 4}$ (where $\nu$ is background viscosity and $\varepsilon^{\prime}$ is the local turbulent dissipation) to large- (basin) scale circulation. In the simulations presented, however, the computational cost of resolving all scales of motion at a high enough buoyancy is prohibitive. As a result, we achieve a compromise by ensuring vertical scales are fully resolved $\left(H / N_{z}<L_{k}\right)$, and horizontal scales are slightly underresolved, such that $L / N_{x}, W / N_{y}<2 L_{k}$ at all points in the domain. We conduct a grid convergence test using a high-resolution test case with grid resolution $\left[N_{x}, N_{y}, N_{z}\right]=[2048,1026,257]$ (such that $L / N_{x}, W / N_{y}, H / N_{z}<L_{k}$ ) and find that there is no significant quantitative difference between the highresolution test case and the simulations presented here. The high resolution achieved by the DNS comes at the cost of a decreased scale separation, which is of order $\sim 10^{4}$ between the turbulent Kolmogorov and Batchelor scales and the basin scale. In the DNS runs, background diffusivity is $\kappa_{\mathrm{DNS}}=2 \times 10^{-6} \mathrm{~m}^{2} \mathrm{~s}^{-1}$ and viscosity is $\nu_{\mathrm{DNS}}=1 \times 10^{-5} \mathrm{~m}^{2} \mathrm{~s}^{-1}$.

An adiabatic and no-slip condition is applied to the southern $(y=0)$ and bottom $(z=0)$ boundaries of the DNS model. The top boundary is subject to a destabilizing buoyancy flux imposed over a region with radius $R=W / 4$ and is free slip. A sponge is imposed in the northern region of the domain (see dotted section in Fig. 1). In the sponge (defined as $W<y<7 W / 5$ ), a Rayleigh damping is imposed with equation $\tau_{s} e^{5[y-(7 / 5) W]}$, where $\tau_{s}$ is a relaxation time scale. At the northern edge of the sponge ( $y=7 \mathrm{~W} / 5)$, temperature, $T$ is damped to a fixed linear stratification profile, $T(z)=\left(N^{2} / g \alpha\right)(z-H / 2)$, vertical and zonal velocities, $w$ and $u$, are free slip, and the meridional velocity $v$ is damped to $d v / d y=0$. The presence of the sponge may indirectly impact the flow development-ideally, the model would be run with a long "buffer" region appended to the northern boundary. However, the DNS runs are extremely computationally expensive, and expanding the size of the model domain would be computationally prohibitive. In addition, the simulations here are primarily investigating transient and quasi-equilibrium mixed layer deepening, which occurs before the large-scale flow features have come into contact with the sponge. As a result, the sponge layer does not significantly impact the results presented here.

\section{2) MOM6}

MOM6 solves the incompressible, hydrostatic NavierStokes momentum equations with a Boussinesq approximation and heat and volume conservation (Adcroft et al. 2019). It is configured with a linear equation of state and beta-plane assumption in a rotating reference frame. The model dimensions are $H=4000 \mathrm{~m}, L=200 \mathrm{~km}$, and $W=$ $200 \mathrm{~km}$. The horizontal grid resolution is $1.25 \mathrm{~km}$, and vertical grid resolution varies exponentially from $2 \mathrm{~m}$ at the surface to $90 \mathrm{~m}$ at the base of the convecting region. In the MOM6 runs, background vertical diffusivity is $\kappa_{\mathrm{MOM} 6}=2 \times 10^{-5} \mathrm{~m}^{2} \mathrm{~s}^{-1}$ and viscosity is $\nu_{\mathrm{MOM} 6}=1 \times$ $10^{-4} \mathrm{~m}^{2} \mathrm{~s}^{-1}$. The southern and bottom boundaries of the model are adiabatic with a quadratic bottom drag, while the top surface is free slip with a surface heat flux imposed over a region with radius $R=W / 4$. The northern boundary of the control volume is open to a region of dimensions $H \times L \times 3 W$ in lieu of a sponge layer (which may indirectly impact the equilibrium state of the flow). The northern boundary of this buffer region has quadratic drag and no heat flux boundary conditions. The MOM6 simulations are run with two boundary layer schemes-KPP and ePBL.

\section{b. Governing parameters}

The flow is governed by several controlling parameters, namely, the flux Rayleigh number $\left(\mathrm{Ra}_{f}\right)$, the Prandtl number $(\operatorname{Pr})$, normalized beta plane $\hat{\beta}$, normalized background stratification $\hat{N}$, and aspect ratios $B$ and $D$ :

$$
\begin{gathered}
\mathrm{Ra}_{f}=\frac{B_{0} H^{4}}{\nu \kappa^{2}}, \quad \operatorname{Pr}=\frac{\nu}{\kappa}, \quad \hat{\beta}=\frac{\beta W}{f_{0}}, \\
\hat{N}=\frac{N}{f_{0}}, \quad B=\frac{L}{W}, \quad D=\frac{H}{W} .
\end{gathered}
$$

During an open-ocean convection event, rotation impacts the horizontal and vertical flow structures in differing ways. As a consequence, rotation is represented by a vertical Rossby number (Ro*), following Maxworthy and Narimousa (1994), and a horizontal Rossby number $\left(\mathrm{Ro}_{L}\right)$, as proposed by Jacobs and Ivey (1999):

$$
\mathrm{Ro}^{*}=\frac{\left(B_{0} / f_{0}^{3}\right)^{1 / 2}}{H}, \quad \operatorname{Ro}_{L}=\left(\frac{B_{0}}{f_{0}^{3} R^{2}}\right)^{1 / 4} .
$$

When Ro* $<0.1$, rotation controls the development of the small-scale turbulent convective plumes triggered by the surface heat flux (Coates et al. 1995). When $\mathrm{Ro}_{L}<0.2$, rotation affects the large-scale horizontal features that arise from the development of a convective chimney (Jacobs and Ivey 1999). Following Jacobs and Ivey $(1998,1999)$, the model runs are conducted in the regime where $\mathrm{Ro}^{*} \geq 0.1$ and $\mathrm{Ro}_{L} \lesssim 0.2$, that is, rotation impacts the large-scale horizontal flow features but does not significantly impact the small-scale turbulent convection in the system. 
TABLE 1. The relevant dimensionless parameters for the suite of simulations conducted in the DNS (cases A-F) and MOM6 (cases 1-5). Two iterations of cases 1-5 are run, once with the KPP mixing scheme and once with the ePBL mixing scheme. Case SO represents the relevant dimensionless parameters for the Southern Ocean. In the DNS runs, $D=2.5 \times 10^{-2}$, and in the MOM6 runs, $D=2 \times 10^{-2}$. In all simulations, $\operatorname{Pr}=5, B=1$, and $\hat{\beta}=0.03$.

\begin{tabular}{|c|c|c|c|c|c|c|c|c|c|}
\hline \multicolumn{5}{|c|}{ Direct numerical simulation (DNS) } & \multicolumn{5}{|c|}{ Modular Ocean Model version 6 (MOM6) } \\
\hline Case & $\mathrm{Ra}_{f}$ & Ro* & $\mathrm{Ro}_{L}$ & $\hat{N}$ & Case & $\mathrm{Ra}_{f}$ & Ro* & $\mathrm{Ro}_{L}$ & $\hat{N}$ \\
\hline A & $1.8 \times 10^{8}$ & 0.24 & 0.15 & 1.83 & 1 & $1.6 \times 10^{21}$ & 0.13 & 0.10 & 4.10 \\
\hline $\mathrm{B}$ & $1.8 \times 10^{8}$ & 0.24 & 0.15 & 4.00 & 2 & $1.6 \times 10^{21}$ & 0.13 & 0.10 & 6.93 \\
\hline $\mathrm{C}$ & $1.8 \times 10^{8}$ & 0.24 & 0.15 & 6.93 & 3 & $1.6 \times 10^{21}$ & 0.13 & 0.10 & 8.90 \\
\hline $\mathrm{D}$ & $9 \times 10^{8}$ & 0.54 & 0.23 & 4.10 & 4 & $1.6 \times 10^{21}$ & 0.13 & 0.10 & 9.80 \\
\hline $\mathrm{E}$ & $9 \times 10^{8}$ & 0.54 & 0.23 & 8.90 & 5 & $1.6 \times 10^{21}$ & 0.13 & 0.10 & 15.50 \\
\hline $\mathrm{F}$ & $9 \times 10^{8}$ & 0.54 & 0.23 & 15.50 & SO & $8 \times 10^{18}-5 \times 10^{20}$ & 0.09 & 0.09 & $0.8-8$ \\
\hline
\end{tabular}

Table 1 shows the relevant governing parameters for the suite of simulations conducted. We run numerical simulations in the DNS (cases A-F) and MOM6 (cases 1-5) with $\hat{N}$ varying from 2 to 15.5 and flux Rayleigh number varying from $O\left(10^{8}\right)$ in the DNS to $O\left(10^{21}\right)$ in the MOM6 runs. The simulations have identical boundary conditions and domains, ensuring that any variations in the flow field will not arise due to a difference in the model setup. Using this simple setup, the mixed layer depth in the large-scale ocean model MOM6 is compared with a turbulence-resolving DNS for the first time. The simulation results are also compared with a theoretical open-ocean convection event in the Southern Ocean, as indicated by case SO in Table 1. We assume an open-ocean convection event in the Southern Ocean of size $R=50 \mathrm{~km}$, with a maximum cooling flux of $800 \mathrm{~W} \mathrm{~m}^{-2}$. Background stratification is assumed to vary over the range $N=10^{-4}-10^{-3} \mathrm{~s}^{-1}$ in the Southern Ocean. Estimates of the dimensional quantities in (2) for the Southern Ocean are taken from Marshall and Schott (1999) and Sohail et al. (2018).

There is approximately an $O\left(10^{10}\right)$ difference in flux Rayleigh number between the Southern Ocean field estimate, which has $\mathrm{Ra}_{f} \sim O\left(10^{19}-10^{20}\right)$, and the DNS, with $\mathrm{Ra}_{f} \sim O\left(10^{8}-10^{9}\right)$. Estimates of $\mathrm{Ra}_{f}$ are obtained for the DNS using $\kappa=2 \times 10^{-6} \mathrm{~m}^{2} \mathrm{~s}^{-1}$, and for the Southern Ocean using $\kappa=5 \times 10^{-5}-2 \times$ $10^{-4} \mathrm{~m}^{2} \mathrm{~s}^{-1}$ following Sohail et al. (2018). As the flux Rayleigh number increases, the dissipation and vertical convective velocity increase. As a consequence, the local Kolmogorov scale $L_{k}$ decreases, and the model grid spacing and time step must be reduced to fully resolve all scales of motion. Increasing the grid resolution and reducing the time step for $\mathrm{Ra}_{f}>10^{9}$ proves to be prohibitively computationally expensive. Therefore, the DNS results presented here have a maximum flux Rayleigh number of $\mathrm{Ra}_{f} \sim 10^{9}$. Nevertheless, we contend that the DNS results are applicable to the Southern Ocean, as the flux Rayleigh number of the simulations is above the critical Rayleigh number,
$\mathrm{Ra}_{c} \sim 10^{7}$, at which turbulent convection is triggered (Julien et al. 1996).

\section{Scaling theory}

We revisit existing scaling theories for mixed layer deepening during open-ocean convection that are derived from the buoyancy balance during the deep convection and lateral exchange and spreading phases. The buoyancy tendency for a fluid at time $t$ is given by

$$
\frac{\partial b}{\partial t}=-\mathbf{u} \cdot \nabla b+\kappa \nabla^{2} b,
$$

where $b=g \alpha T^{*}$ and $T^{*}$ is the temperature deviation from the initial state, that is, $T^{*}=T-T(t=0)$.

Initially, the buoyancy forcing is communicated to the interior through a diffusive sublayer (Turner 1973). The dominant balance controlling the depth of the diffusive sublayer is

$$
\frac{\partial b}{\partial t} \sim \kappa \nabla^{2} b
$$

which scales as

$$
\frac{\Delta b}{t} \sim \kappa \frac{\Delta b}{\delta^{2}},
$$

where $\delta$ is the diffusive sublayer depth. Solving Eq. (5) for $\delta$, we obtain a diffusive sublayer depth, which scales as

$$
\delta \sim \sqrt{\kappa t} .
$$

The diffusive sublayer grows in the initial stages of open-ocean convection until it is overtaken by the deepening of the turbulent convective mixed layer. In nonpenetrative convection, Turner (1973) proposed the following buoyancy balance:

$$
\frac{\partial b}{\partial t}=\frac{B_{0}}{g \alpha} h
$$




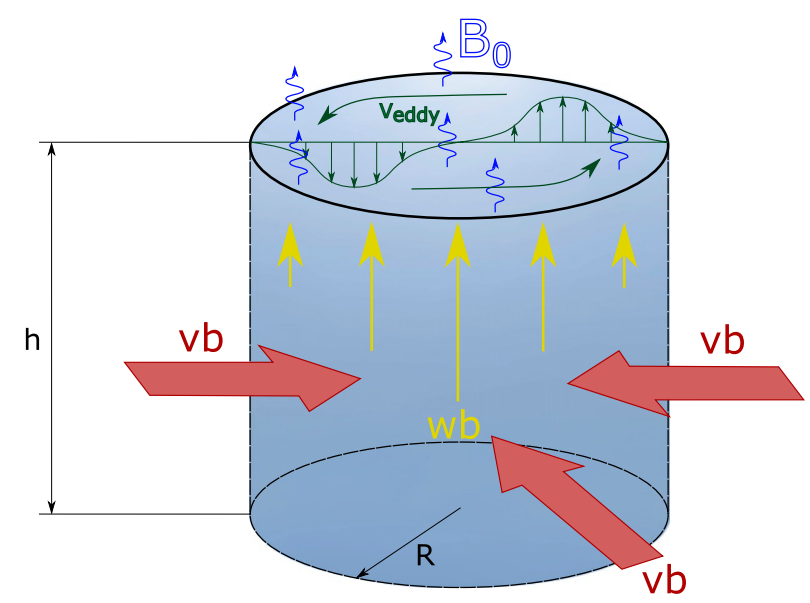

FIG. 2. A schematic representation of the mixed patch during open-ocean convection, visualized as a uniform cylinder of mixed layer depth $h$ and radius $R$. Surface buoyancy fluxes are shown in blue, the velocity profile of the geostrophic current is shown in green, the large-scale eddy fluxes $\left.\overline{v^{\prime} b^{\prime}}\right|_{E}$ are shown in red, and the turbulent vertical buoyancy fluxes $\left.\overline{w b^{\prime}}\right|_{T}$ are shown in yellow.

where $h$ is the depth of the turbulent convective mixed layer. This balance assumes there is no entrainment at the base of the mixed layer, and convection primarily acts to neutralize the buoyancy gradient in the fluid. Assuming the vertical buoyancy difference can be approximated as $\Delta b \sim N^{2} h$, we obtain the classical result of mixed layer depth over time:

$$
h(t)=\Lambda\left(\frac{B_{0} t}{N^{2}}\right)^{1 / 2}
$$

where $\Lambda$ is a constant found to be $\sqrt{2}$ by Turner (1973).

At steady state, the buoyancy tendency $\partial b / \partial t$ is zero, so the prevailing balance is between the surface heat flux $B_{0}$ and lateral large-scale eddy fluxes $\left.\overline{v^{\prime} b^{\prime}}\right|_{E}$. For simplicity, we define a cylindrical control volume with a radius $R$ equal to the radius of the cooling patch and a height $h$ equal to the mixed layer depth, as shown in Fig. 2. We also assume that advection dominates diffusion after the onset of convection (Visbeck et al. 1996; Jones and Marshall 1997). Therefore, upon integrating over the cylindrical control volume, the buoyancy budget at steady state becomes

$$
\int B_{0} d S=\oint \int v b \cdot \hat{n} d l
$$

where $S$ is the top surface of the cylinder. Equation (9) scales as

$$
\pi R^{2} B_{0} \sim 2 \pi R h V_{\text {eddy }} \Delta b,
$$

where $V_{\text {eddy }}$ is the eddy velocity, shown schematically in Fig. 2 . The eddy velocity $V_{\text {eddy }}$ is assumed to scale with the geostrophic current velocity $U_{g}$ (Visbeck et al. 1996), which in turn is dependent on the buoyancy gradient $\Delta b$ such that

$$
V_{\text {eddy }} \sim U_{g} \sim \frac{\Delta b h}{\mu f}
$$

where $\mu$ is the horizontal scale of the eddies in the system. At steady state, $\mu$ can be approximated as the mixed layer Rossby deformation radius, $\mu=N h / f$.

The lateral buoyancy difference $\Delta b$ scales as $\Delta b=$ $N^{2} h$, as discussed above. Plugging Eq. (11) and the relevant assumptions for $\mu$ and $\Delta b$ into Eq. (10), we obtain the final depth of the mixed layer $h_{\text {final }}$ as a function of externally controlled parameters:

$$
h_{\text {final }}=\gamma \frac{\left(B_{0} R\right)^{1 / 3}}{N}
$$

where $\gamma$ is an empirical constant. The term $\gamma$ is obtained from the turbulence-resolving DNS runs, and is assumed to apply for all cases, allowing us to compare directly between the DNS and MOM6 runs. The quasi-equilibrium mixed layer depth prediction in Eq. (12) is well established (Visbeck et al. 1996; Legg et al. 1996; Jones and Marshall 1997), as is the scaling for eddy velocity (Fox-Kemper et al. 2008). Combining the transient mixed layer deepening scaling in Eq. (8) with the quasi-equilibrium scaling in Eq. (12), we obtain a prediction for the time at which the transition from vertical buoyancy transport to horizontal buoyancy transport occurs, from Visbeck et al. (1996):

$$
t_{\text {final }}=\Omega\left(\frac{R^{2}}{B_{0}}\right)^{1 / 3},
$$

where $\Omega$ is an empirical constant.

For ease of comparison between the DNS output, MOM6 results and the Southern Ocean, key variables are nondimensionalized such that $\hat{t}=t N, \hat{b}=b /\left(B_{0} R N^{3}\right)^{1 / 3}$, $[\hat{u}, \hat{v}]=\left[u /\left(B_{0} R\right)^{1 / 3}, v /\left(B_{0} R\right)^{1 / 3}\right], \quad \hat{w}=w N^{1 / 3} /\left(B_{0}^{4} R\right)^{1 / 9}$ [from the scaling $w \sim\left(B_{0} h\right)^{1 / 3}$ ] and $\hat{\kappa}_{v}=\kappa_{v} / \kappa$, where a hat denotes a nondimensional quantity.

\section{Results}

A central focus of this work is to understand deep convection over a wide parameter space and to improve existing large-scale models by comparing the DNS output directly with the large-scale MOM6 simulations. To this end, we compare the flow solutions, mixed layer deepening and vertical diffusivity in both models and identify potential issues in the 

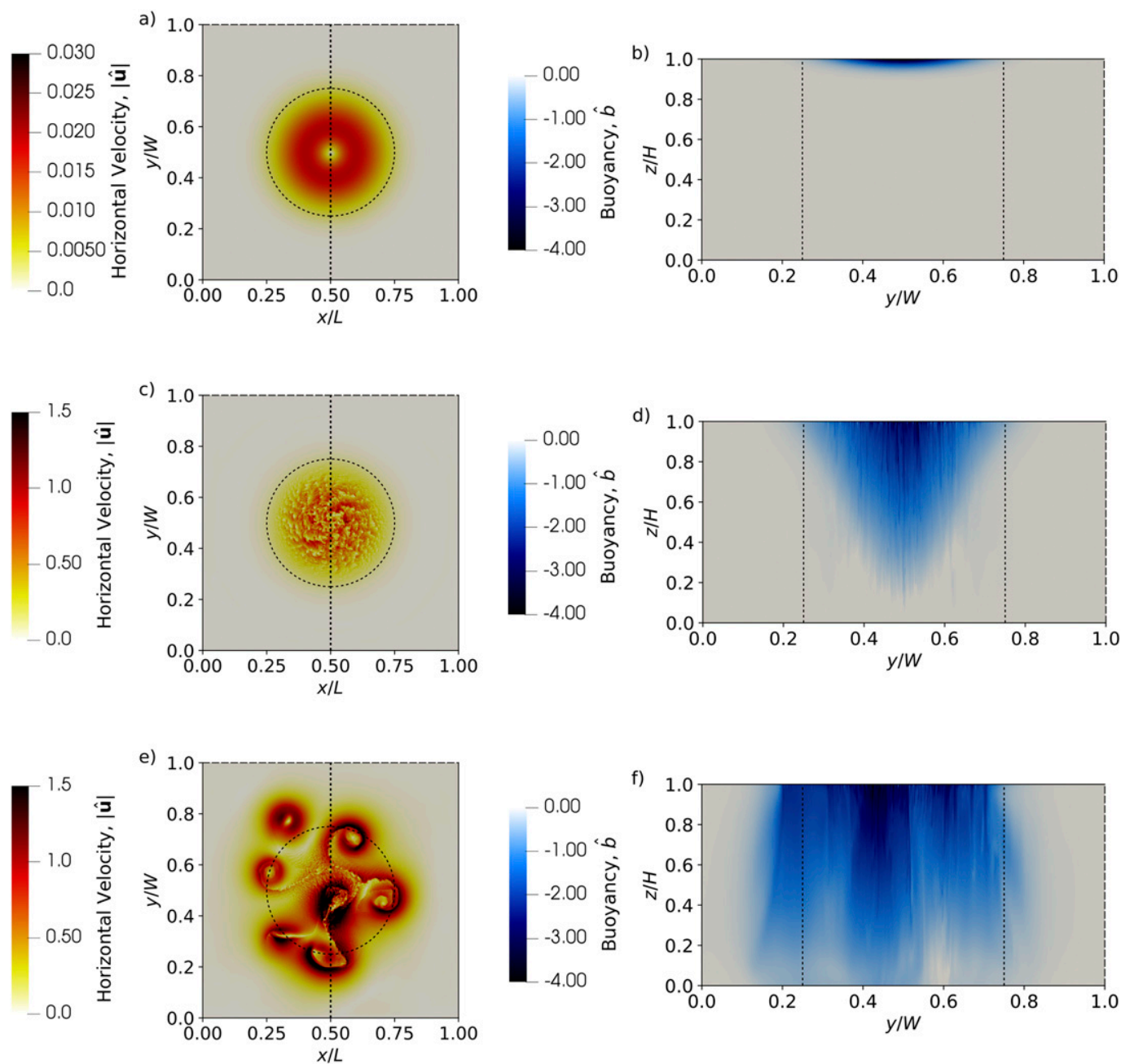

FIG. 3. Flow solutions over a range of times for case D. (a),(c),(e) Normalized root-mean-square horizontal velocity $|\hat{\mathbf{u}}|=\left|\sqrt{\hat{u}^{2}+\hat{v}^{2}}\right|$ in the $x-y$ plane at $z / H=0.98$ for times $\hat{t}=13.4,102.4$, and 355.9, respectively. (b),(d),(f) Normalized buoyancy $\hat{b}$ in the $y-z$ plane at $x / L=0.5$ for times $\hat{t}=13.4,102.4$, and 355.9, respectively. The dotted vertical lines in (a), (c), and (e) indicate the location of the plane shown in (b), (d), and (f). Dotted circles in (a), (c), and (e) and dotted vertical lines in (b), (d), and (f) indicate the horizontal extent of the destabilizing buoyancy signal. Dashed lines indicate the presence of a sponge at $y / W>1$. Note varying velocity scales between figures.

characterization of open-ocean convection in largescale ocean models.

\section{a. Flow solutions}

The DNS and MOM6 simulations both exhibit the three distinct stages of open-ocean convection that have been documented previously: diffusion, deep convection, and baroclinic instability and lateral spreading. Figure 3 shows the normalized horizontal velocity field $\hat{u}$ inside the boundary layer (at $z / H=0.98$ ) and the buoyancy $\hat{b}$ through depth (at $x / L=0.5$ ) during each phase of open-ocean convection in the DNS (case D). During the diffusion phase, the sensible heat input is diffusively transferred downgradient, resulting in a buoyancy field that closely matches the imposed
Gaussian surface flux forcing. The lateral buoyancy gradient triggers the development of a geostrophic current (Cui and Street 2001), as shown at $\hat{t}=13.4$ in Fig. 3a. Due to the radial variance in the buoyancy forcing, the geostrophic current exists throughout the cooling patch, rather than as a rim current [as would be the case for a "cooling disk," such as in Maxworthy and Narimousa (1994)]. The strength of the geostrophic current is proportional to the lateral gradient of the surface buoyancy forcing, so the current velocity is strongest where the lateral buoyancy gradient is highest, at $W / 8$ from the center of the cooling patch. The region of destabilizing buoyancy driven by diffusion is largely surface-isolated and reaches a depth of $z / H \approx 0.92$, from Fig. $3 b$. 
The vertical advective component of turbulent convection quickly outpaces the growth of the diffusive mixed layer, and by $\hat{t}=102.4$ a rich field of small-scale convective plumes has developed in the DNS model during the deep convection phase (Figs. 3c,d). The largescale geostrophic current interacts with small-scale turbulence, resulting in a small-scale horizontal velocity field that rotates the cooling patch (Fig. 3c). The convective plumes are largely concentrated in the region of destabilizing buoyancy forcing and penetrate at a depth proportional to the strength of the surface buoyancy forcing. Therefore, the penetration depth of the convective plumes decreases radially outwards from the center of the cooling patch (Fig. 3d). The presence of turbulence in the vertical convection is also readily apparent from the variability in the buoyancy field in Fig. 3d.

Over time, the lateral buoyancy gradient increases and triggers baroclinic instability in the baroclinic instability and lateral spreading phase. Upon the development of baroclinic eddies, the horizontal velocity field, shown in Fig. $3 \mathrm{e}$ at $\hat{t}=355.9$, deforms the symmetric nature of the convective patch. The flow at this stage is characterized by features that span a range of scales of motion, from small-scale turbulence to largescale geostrophic currents. Figure $3 \mathrm{f}$ shows that the negative buoyancy region extends beyond the horizontal extent of the surface buoyancy forcing (shown by the vertical dotted lines in Figs. 3b,d,f). The $y-z$ plane in Fig. 1 shows the total buoyancy field (calculated with total temperature $T$ rather than temperature deviation $T^{*}$ ) during the lateral spreading phase in the DNS, at $\hat{t}=355.9$ in case $\mathrm{D}$. The background linear stratification is well mixed in the region of cooling, with isopycnals (shown as white contours) outcropping along the fringes of the convecting patch where the lateral buoyancy gradient is strongest. The turbulent convective plumes shown in the vertical velocity field in the $z-x$ plane in Fig. 1 penetrate density surfaces, leading to the vertical mixing of buoyancy seen in the $y-z$ plane.

Figure 4 shows the simulated flow field in the MOM6 simulation for case 1 during each phase of open-ocean convection (slices are taken at the same spatial locations as the DNS results in Fig. 3). The azimuthal geostrophic current and diffusive boundary layer are evident in the MOM6 output during the diffusion phase at $\hat{t}=22.1$, and closely align with the results from the DNS output (see Figs. 4a,b). During the deep convection phase, the MOM6 results, illustrated in Figs. $4 \mathrm{c}$ and $4 \mathrm{~d}$ at $\hat{t}=93.0$, deviate significantly from the DNS. The shape of the mixed patch is Gaussian and similar to the DNS results (see Fig. 4d compared with Fig. 3d). However, the penetration depth of the mixed patch is quite different between the MOM6 runs and the DNS, as it is a function of the strength of the surface forcing and the background stratification. In addition, the vertical convective plumes lack any degree of turbulence, and grow in a laminar, nonpenetrative fashion in the MOM6 simulation. The laminar nature of the convection is illustrated by the horizontal velocity field in Fig. 4c, where the signature of small-scale, turbulent convection is entirely absent, and the horizontal geostrophic current has not been modified by turbulence. The three-dimensionality of the flow is therefore not represented in the MOM6 results during the deep convection phase of the flow. Upon the evolution of the resolved baroclinic eddies in the baroclinic instability and lateral spreading phase, the MOM6 output once again resembles the turbulence-resolving DNS, as illustrated by Figs. $4 \mathrm{e}$ and $4 \mathrm{f}$ at $\hat{t}=978.3$. The lateral flux of the convective patch by baroclinic eddies introduces some three-dimensionality to the flow, with vertical convection and lateral eddies acting in concert at quasi-equilibrium. However, the small-scale turbulence seen in the DNS is still not present in the MOM6 simulation at quasi-equilibrium.

The flow features observed in the MOM6 simulation and DNS align with the typical phases of open-ocean convection in high-latitude regions of the ocean (Marshall and Schott 1999). In particular, the lateral spreading of eddies in the simulations looks qualitatively similar to multiple numerical and laboratory studies of open-ocean convection (e.g., Ivey et al. 1995; Visbeck et al. 1996; Jones and Marshall 1997; Cui and Street 2001; Noh et al. 2003; Gelderloos et al. 2011). However, the MOM6 model differs from the DNS output during the deep convection phase, resulting in qualitatively different flow features associated with the turbulence field. The results shown in Fig. 4 are with the ePBL boundary layer parameterization. That said, the flow solutions (and in particular the representation of turbulence during the deep convection phase) do not vary significantly when employing the KPP boundary layer parameterization (not shown).

\section{b. Mixed layer depth}

We investigate mixed layer deepening during openocean convection by diagnosing the mixed layer depth over time and comparing the transient and quasiequilibrium depth between the simulations. The choice of mixed layer depth criterion in ocean models has long been the subject of debate. A large proportion of global ocean modeling studies diagnose mixed layer depth based on a threshold criterion, commonly a threshold in the density difference between the ocean surface and base of the mixed layer. However, Holte and Talley (2009), and more recently, Courtois et al. (2017) argue that threshold criteria contribute to the anomalously 

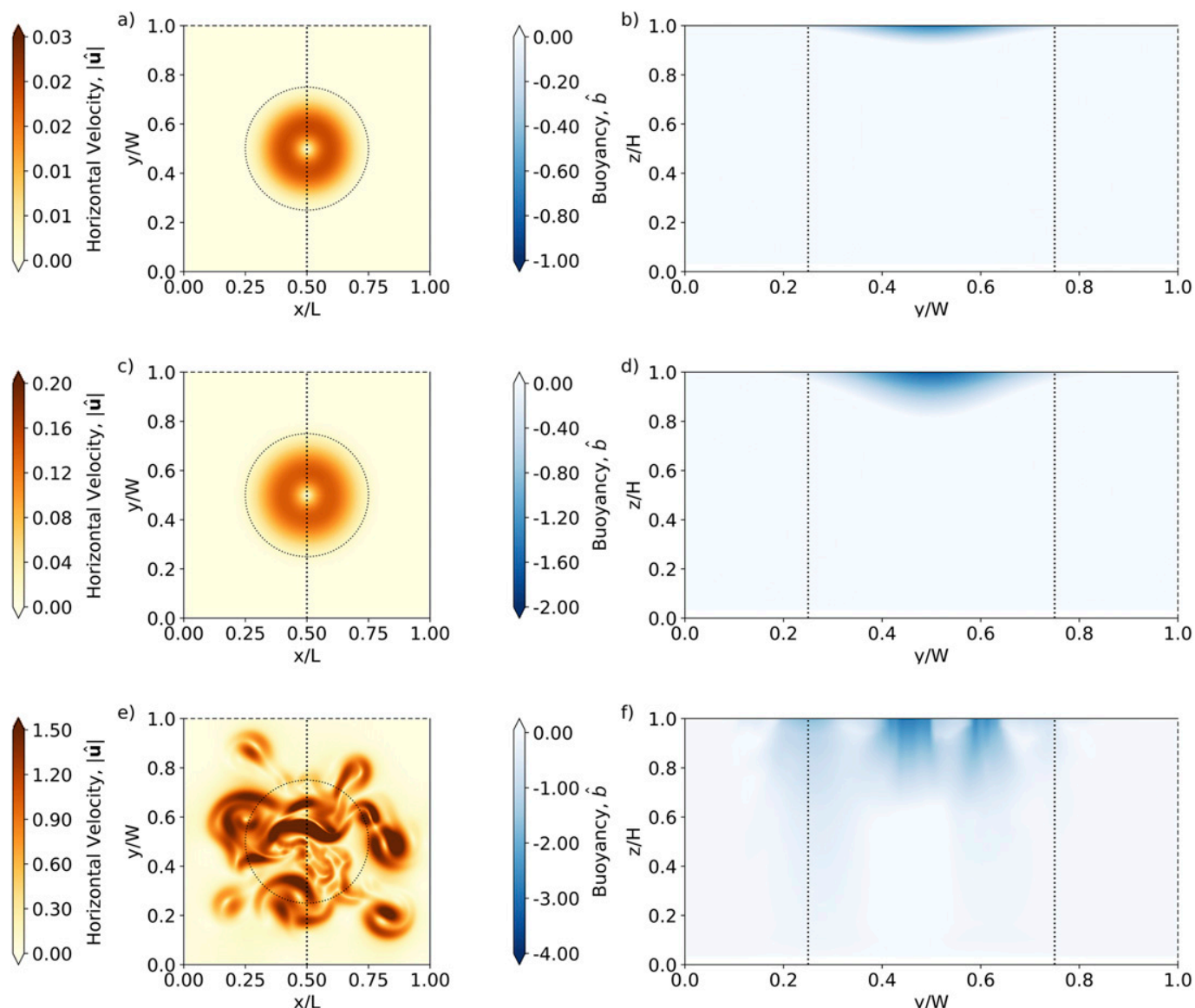

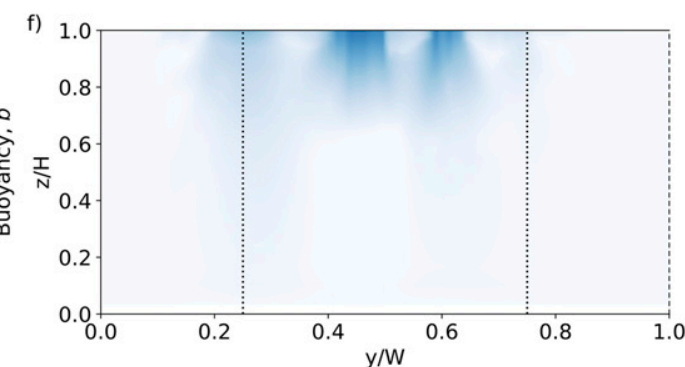

FIG. 4. Flow solutions over a range of times for case 1 with the ePBL mixing scheme. (a),(c),(e) Normalized rootmean-square horizontal velocity $|\hat{\mathbf{u}}|=\left|\sqrt{\hat{u}^{2}+\hat{v}^{2}}\right|$ in the $x-y$ plane at $z / H=0.98$ for times $\hat{t}=22.1,93.0$, and 978.3, respectively. (b),(d),(f) Normalized buoyancy $\hat{b}$ in the $y-z$ plane at $x / L=0.5$ for times $\hat{t}=22.1,93.0$, and 978.3, respectively. The dotted vertical lines in (a), (c), and (e) indicates the location of the plane shown in (b), (d), and (f). Dotted circles in (a), (c), and (e) and dotted vertical lines in (b), (d), and (f) indicate the horizontal extent of the destabilizing buoyancy signal. Dashed lines indicate the presence of a sponge at $y / W>1$. Note varying velocity scales between figures.

deep mixing seen during deep convection in ocean models. Currently, there is no consistent choice of mixed layer criterion across modeling studies.

Given the inherent differences between the DNS and MOM6 models, it is imperative to use mixed layer depth criteria that are comparable between the two models. The choice of mixed layer depth criterion in the DNS is physically motivated. We assume that the mixed layer lies at the approximate depth where the horizontally averaged vertical buoyancy transport $w b$ is close to zero based on the dominant terms of the buoyancy budget in Eq. (3). Specifically, mixed layer depth is defined as the depth at which $1 / L W \iint w b d x d y=0.01 B_{0}$. The choice to set the criterion as $1 \%$ of $B_{0}$ is motivated by the need to capture the full vertical extent of the mixed layer without picking up the significant variability in the bottommost part of the mixed layer.
Many vertical processes are parameterized in the MOM6 simulations, so explicitly calculating mixed layer depth in the same way as the DNS is not possible. In the absence of an equivalent definition to the DNS, and given the concerns raised by prior research regarding the density threshold criterion, we use the mixed layer depths calculated by the KPP and ePBL mixing schemes in the MOM6 simulations. To ensure the mixed layer depths in the MOM6 simulations are comparable to the DNS, we take a probability density function (PDF) of the MOM6 mixed layer depths within the cylindrical control volume defined by Fig. 2, and pick a percentile that corresponds to the $1 \%$ threshold used in the DNS. The DNS mixed layer depth is obtained from horizontally averaging $w b$ over the whole domain, while the MOM6 mixed layer depth is obtained by taking a PDF over a cylindrical control volume. Therefore, to obtain a 
a)

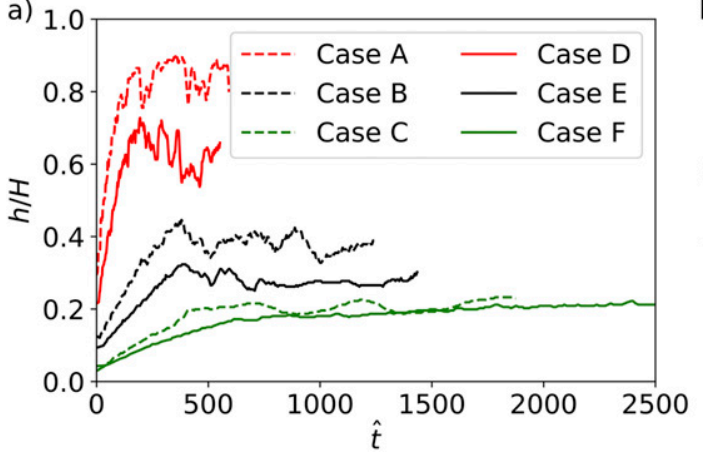

b)

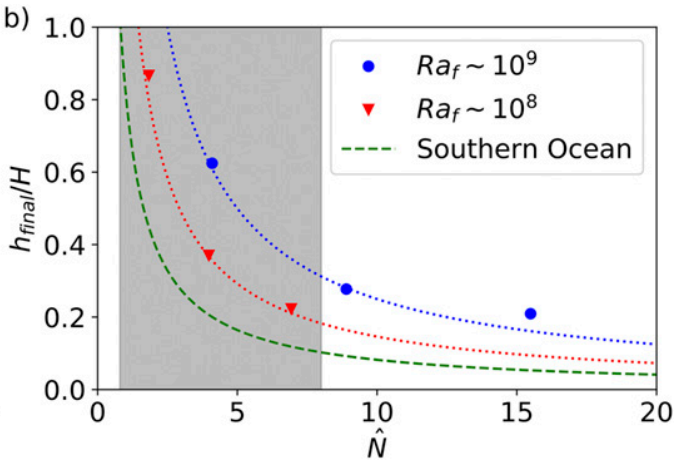

FIG. 5. (a) Mixed layer depth over time for the DNS simulations conducted. Solid lines correspond to the highflux Rayleigh number $\left(\mathrm{Ra}_{f} \sim 10^{9}\right)$ runs. Dashed lines correspond to the low-flux Rayleigh number $\left(\mathrm{Ra}_{f} \sim 10^{8}\right)$ runs. (b) Quasi-equilibrium mixed layer depths for the DNS simulations, calculated as the time-averaged mixed layer depth over the final $\Delta \hat{t}=[80,188,333,94,214,539]$ for cases $A-F$, respectively. Dotted lines indicate the theoretical mixed layer depth predictions from Eq. (12) for the DNS runs with $\gamma=1.76$, calculated by curve fitting with the model output. The dashed line indicates the scaling prediction from Eq. (12) for the Southern Ocean with $\gamma=$ 1.76. The gray box highlights the range of background stratifications relevant to the Southern Ocean.

mixed layer depth at the same approximate depth level as the DNS, we assign the mixed layer depth in the MOM6 simulations to be the depth in the $100-1 \times$ $\left(\pi R^{2} / L W\right)$ percentile of the PDF of all mixed layer depths. For the parameters used in the present simulations, we obtain mixed layer depth in the 94.9th percentile of the PDF.

Figure 5a shows the mixed layer depth over time for the suite of DNS simulations conducted, and Fig. 5b shows the final mixed layer depth for the suite of DNS simulations. In the turbulence-resolving simulations, the mixed layer broadly follows the scaling theory derived by Turner (1973), $h \sim t^{1 / 2}$. As predicted by the scaling theory in Eq. (8), increasing the background stratification $\hat{N}$ results in a reduced rate of mixed layer deepening, while increasing the imposed buoyancy $B_{0}$ increases the rate of deepening (see Fig. 5b). Overall, the transient mixed layer growth shows significant internal variability, likely due to the nonlinear nature of convection.

At quasi-equilibrium, strong lateral eddy fluxes arrest the growth of the mixed layer, resulting in a final mixed layer depth $h_{\text {final }}$. The DNS output closely matches the scaling predictions from Eq. (12) with $\gamma=1.76$, calculated by curve fitting with the model output and shown by the dotted lines in Fig. 5b. As predicted by the scaling, increasing background stratification inhibits the growth of the turbulent mixed layer, resulting in shallower quasi-equilibrium mixed layer depths. In addition, increasing the buoyancy (represented by the flux Rayleigh number, $\mathrm{Ra}_{f}$ ) results in a deeper penetration and quasi-equilibrium depth. These results validate widely used, existing scaling theories of open-ocean convection, providing confidence in incorporating these scaling predictions in future models.
The validated scaling predictions can be compared with the mixed layer deepening in analogous MOM6 results, shown in Fig. 6. Dotted lines in Fig. 6 correspond to the scaling predictions from Eqs. (8) and (12), with $\Lambda=0.07$ and $\gamma=1.76$. The coefficient $\gamma$ is obtained from the DNS results, and the coefficient $\Lambda$ is calculated by curve fitting with the MOM6 output during the transient phase of mixed layer growth. The value of $\Lambda$ does not vary significantly between mixed layer parameterization schemes. As with the DNS, the background stratification plays a leading-order role in determining the depth over which convective deepening occurs in the MOM6 results. As background stratification increases from case 1 to 5 , the convection is confined to a shallower region. In all cases, the transient mixed layer deepening follows the canonical $h \sim t^{1 / 2}$ scaling, following the DNS results. This trend is evident in both mixed layer parameterizations. However, in contrast to the DNS, the deepening of the mixed layer is smooth-there is relatively little internal variability to the flow. The lack of internal variability in the mixed layer growth is consistent with the relatively laminar deepening of the mixed layer in Figs. $4 \mathrm{~b}$ and $4 \mathrm{c}$.

As the mixed layer deepens, it "overshoots," becoming anomalously deep before thinning again at quasi-equilibrium (see peaks in mixed layer depth in Fig. 6). This overshoot is not observed in the turbulent DNS results (Fig. 5a) and occurs during the deep convection phase of the flow for all mixed layer parameterizations. Note that the overshoot is higher for the KPP mixed layer parameterization compared with the ePBL, particularly in the weakest stratification case (case 1). We posit that the overshoot seen in the MOM6 simulations is a consequence of the lack of representation of finer 
a)

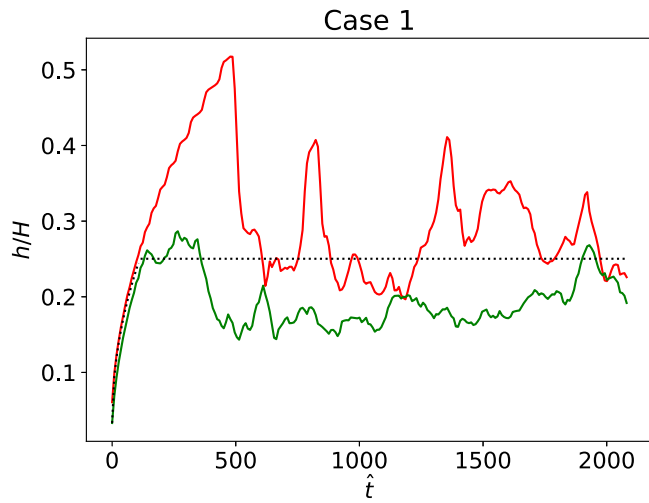

c)

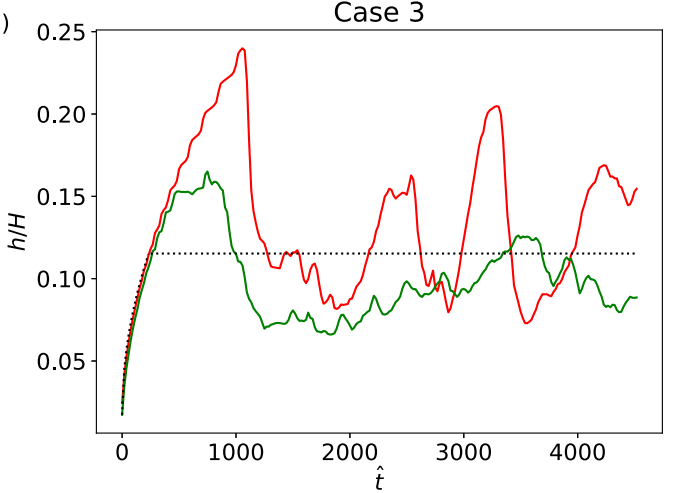

e)

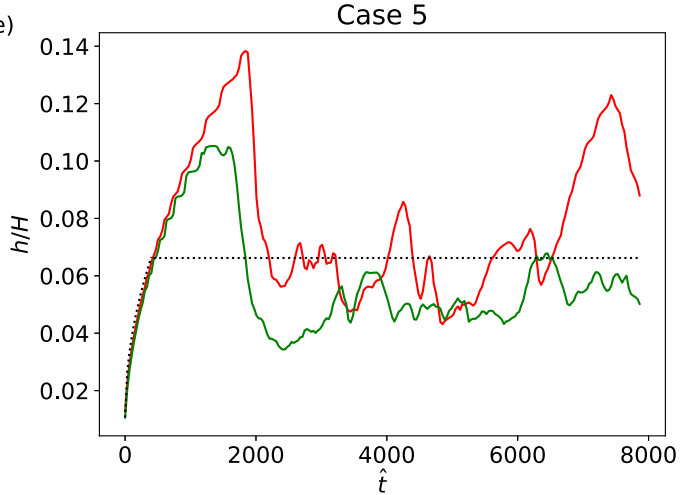

b)

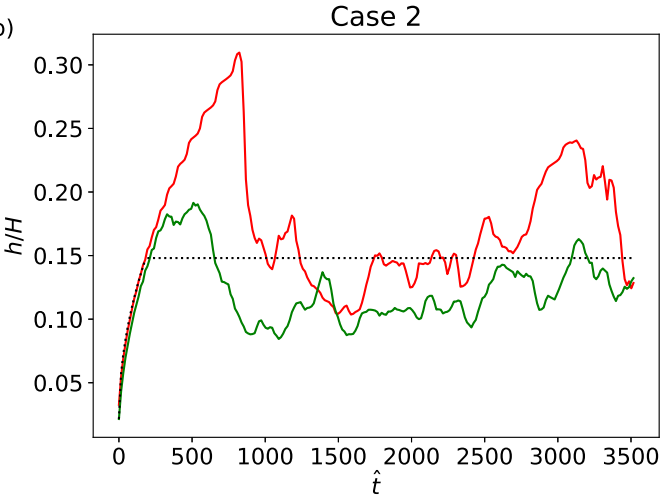

d)

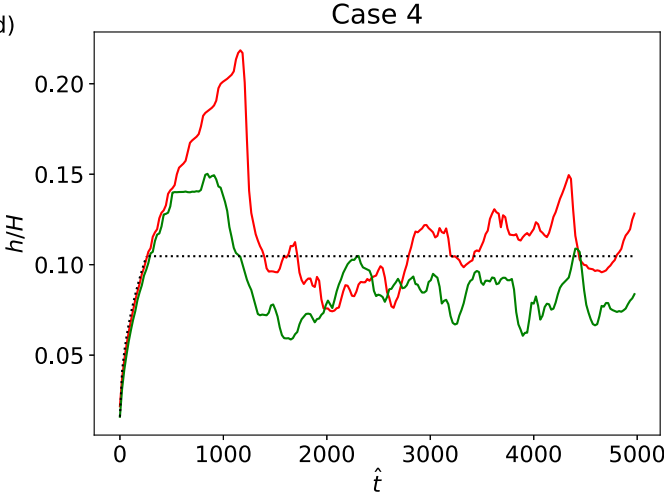

FIG. 6. Mixed layer depth over time for the MOM6 simulations conducted. Dotted lines correspond with the scaling prediction for mixed layer deepening and quasi-equilibrium depth, calculated from Eqs. (8) and (12), with $\Lambda=0.07$ and $\gamma=1.76$. Note the difference in $x$ - and $y$-axis ranges between figures.

scales of turbulence in the model. The turbulent variability in the convective plumes destabilizes the geostrophic azimuthal current, hastening the growth of baroclinic eddies when turbulence is resolved. In the MOM6 configuration, baroclinic eddies are being triggered later, leading to the observed overshoot in mixed layer depth before quasi-equilibrium.

Figure 7 shows the final mixed layer depth over the range of background stratifications run in MOM6. The quasi-equilibrium mixed layer depth $h_{\text {final }}$ follows the scaling predictions laid out in Eq. (12), as shown in
Fig. 7a. The close alignment between the MOM6 simulations and the scaling is consistent regardless of the mixed layer parameterization employed (see Fig. 7b). Thus, despite large inaccuracies in transient mixed layer deepening, the equilibrium depth is relatively robust. An analysis of quasi-equilibrium and transient mixed layer depths was also conducted with the density threshold mixed layer depth criterion. The mixed layer depths calculated from the simple density threshold were significantly deeper than the scaling predictions for all cases at all stages of mixed layer 

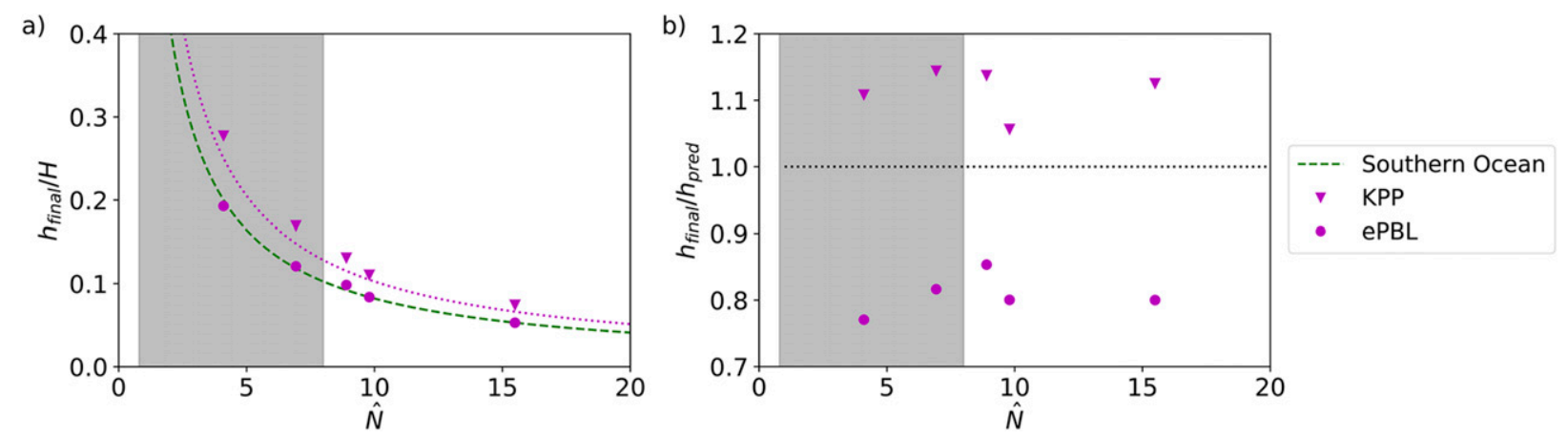

FIG. 7. (a) Quasi-equilibrium mixed layer depth for the suite of MOM6 simulations conducted. The dotted magenta and dashed green lines correspond to the theoretical scaling prediction from Eq. (12) for the MOM6 runs and the Southern Ocean, respectively, with $\gamma=1.76$ from the DNS results. Quasi-equilibrium mixed layer depth is calculated by time-averaging over the final $\Delta \hat{t}=[1107,1871,2403,2645,4183]$ for cases 1-5, respectively. (b) Quasi-equilibrium mixed layer depth normalized by the theoretical scaling prediction from Eq. (12). The gray box highlights the range of background stratifications relevant to the Southern Ocean.

growth. This finding aligns with prior work by Holte and Talley (2009) and Courtois et al. (2017). Alternative mixed layer depth definitions were not tested in this analysis.

\section{c. Vertical diffusivity}

Convective adjustment schemes largely rely on instantaneously increasing diffusion to enhance mixing in unstable regions of the domain. Therefore, quantifying the vertical diffusivity during open-ocean convection can be instructive in guiding future convective parameterizations. Figure 8a shows the inferred vertical diffusivity [calculated as $\kappa_{v}=-(\overline{w b}) /(\partial b / \partial z)$ in the cylindrical control volume defined by Fig. 2] for the DNS simulations. The initiation of convection results in the enhancement of vertical buoyancy fluxes $w b$ and the reduction of the vertical buoyancy gradient $\partial b / \partial z$ leading to a peak in vertical diffusivity during the deep convection phase of flow development. Note that while the vertical buoyancy gradient is eliminated in the majority of the convecting region [following the simple convective deepening model of Turner (1973)], the vertical buoyancy gradient is nonzero in the nearsurface and near-bottom parts of the convecting cylinder. Therefore, in a volume-averaged sense the vertical buoyancy gradient reduces during vertical convection but does not quite reach zero. Upon the initiation of baroclinic instability, lateral buoyancy fluxes dominate the flow and the coherent, homogeneous mixed region is broken up. Therefore, vertical buoyancy fluxes drop, leading to the observed decrease in vertical diffusivity in the DNS model. The final diffusivity in the model depends on the imposed background stratification, with higher quasi-equilibrium diffusivity at weaker background stratifications (Fig. 8b).

Figure 9 shows the vertical diffusivity in the MOM6 runs over time, calculated as the volume-averaged diffusivity inside the cylindrical control volume illustrated in Fig. 2. The vertical diffusion follows a pattern consistent with the DNS output (as shown in Fig. 8), with a peak in diffusion during deep convection followed by a drop to a stable quasi-equilibrium value. The peak in diffusion varies based on the background stratification
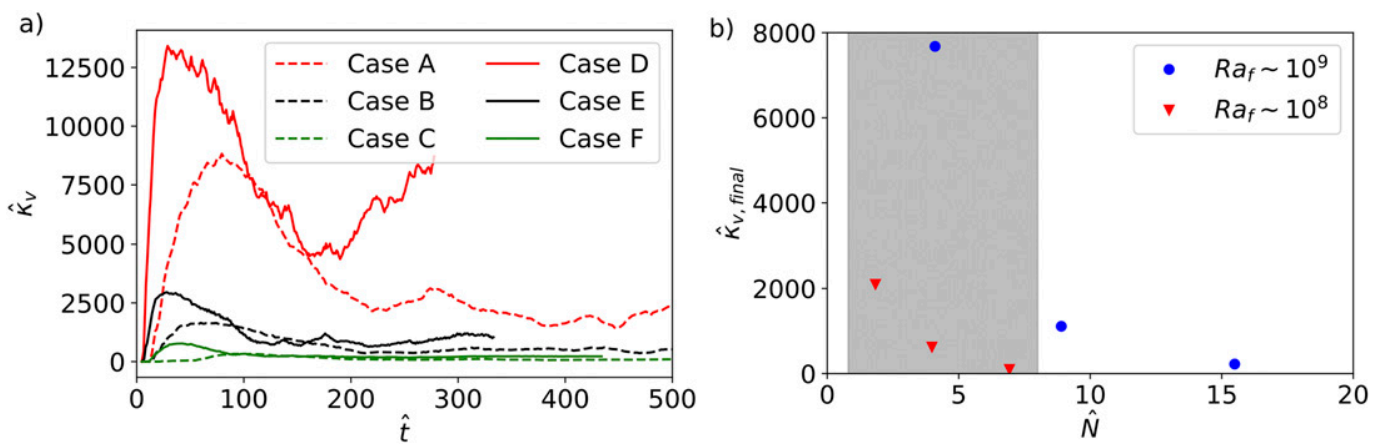

FIG. 8. Normalized vertical diffusivity $\hat{\kappa}_{v}$ (a) over time and (b) at quasi-equilibrium, for the DNS runs conducted. Solid lines correspond to the high-flux Rayleigh number $\left(\operatorname{Ra}_{f} \sim 10^{9}\right)$ runs. Dashed lines correspond to the low-flux Rayleigh number $\left(\mathrm{Ra}_{f} \sim 10^{8}\right)$ runs. The gray box highlights the range of background stratifications relevant to the Southern Ocean. 

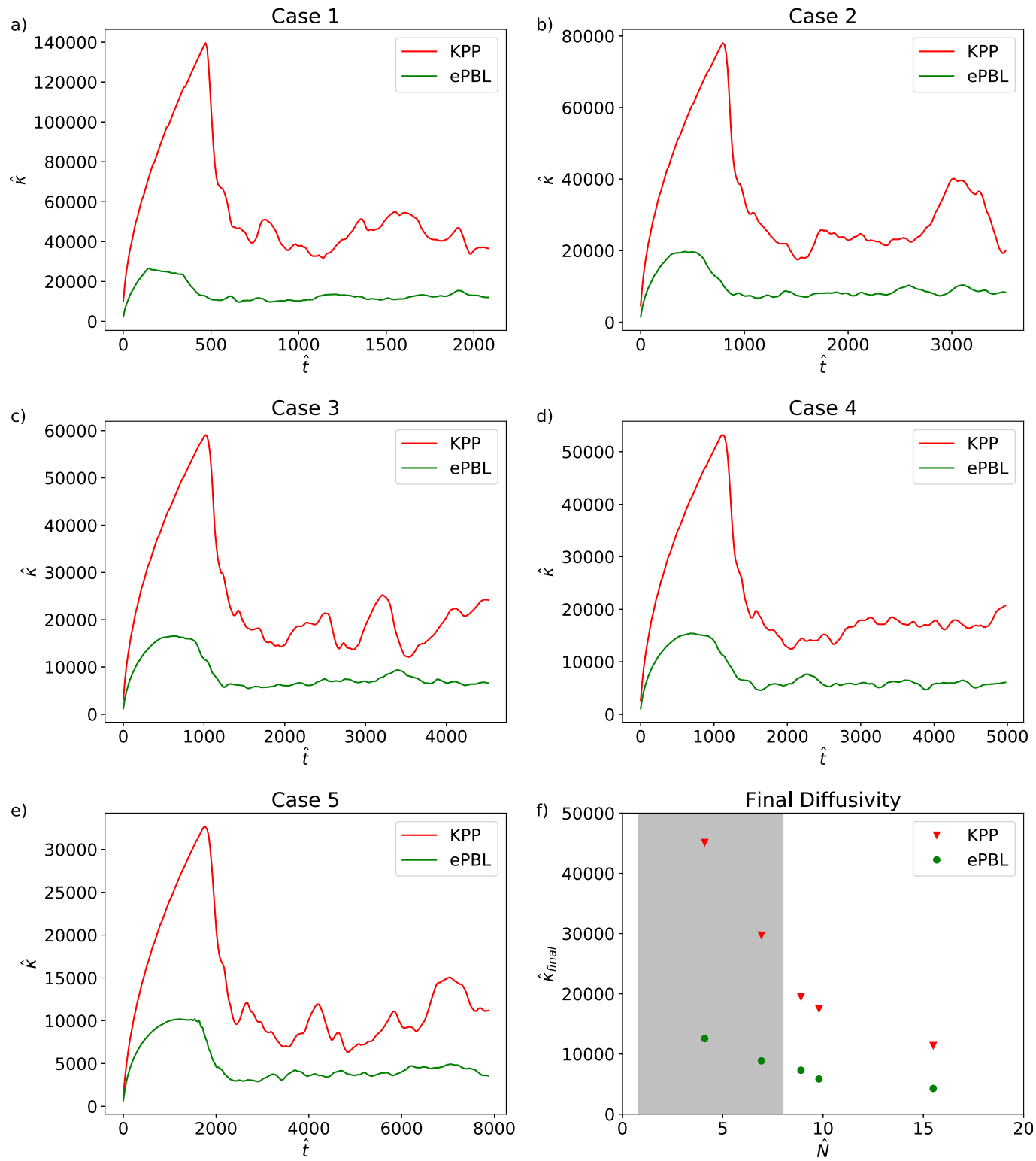

FIG. 9. Normalized diffusivity $\hat{\kappa}$ (a)-(e) over time and (f) at quasi-equilibrium, for the suite of MOM6 simulations conducted. The gray box in (f) highlights the range of background stratifications relevant to the Southern Ocean. Note the difference in $x$ - and $y$-axis ranges between panels.

and mixing scheme used in the model. At all stratifications, the peak in vertical diffusion is highest in the KPP configuration (Figs. 9a-e). The quasi-equilibrium value of diffusion also varies based on the background stratification and mixing scheme. Closely following the DNS results in Fig. 8b, the MOM6 quasi-equilibrium diffusion is highest in the weakly stratified cases and decreases with increasing background stratification (see Fig. 9f). The simulations with the ePBL mixing scheme have a lower quasi-equilibrium diffusion. Interestingly, the difference in quasi-equilibrium diffusivity between the
KPP and ePBL schemes increases at weaker stratifications (Fig. 9f). In spite of this, the difference in quasiequilibrium mixed layer depths between the KPP and ePBL schemes remains approximately the same over the range of background stratifications studied (Fig. 7b). The decoupling between final diffusivity and mixed layer depth indicates that the final diffusivity has little bearing on the quasi-equilibrium state of the system. This is primarily because, at quasi-equilibrium, lateral fluxes due to mixed layer eddies set the depth of the mixed layer. Vertical processes, which are represented 
by vertical diffusion, do not play a significant role. Therefore, there is relatively little variance in the difference in final mixed layer depth between the KPP and ePBL schemes, despite the increasing spread in diffusion at weaker stratifications in Fig. 9f.

\section{Discussion and conclusions}

We compare the representation of open-ocean convection in a turbulence-resolving DNS and large-scale ocean model (MOM6). The DNS captures all scales of motion (albeit across a narrow range of length scales), allowing for a comprehensive review of the physical processes at work during open-ocean convection. We endeavor to keep the DNS and MOM6 simulations easily comparable by maintaining an identical model setup and boundary conditions where computationally possible. Multiple prior studies have used slightly different model configurations, making a direct comparison difficult. For instance, Maxworthy and Narimousa (1994) and Send and Marshall (1995), among others, explored deep convection in an unstratified system, while Cui and Street (2001) explored deep convection with the presence of slope topography. These seemingly minor tweaks to the boundary conditions result in significant changes to the flow dynamics. Therefore, it is difficult to directly compare the setup and forcing parameters chosen here to prior research. That said, the open-ocean convection event simulated in the DNS does share similarities with prior numerical and laboratory studies of open-ocean convection (Visbeck et al. 1996; Jones and Marshall 1997; Legg et al. 1998; Marshall and Schott 1999; Cui and Street 2001; Gelderloos et al. 2011).

In the DNS, the initial growth of the mixed layer is largely diffusive, and quickly gives way to vigorous, three-dimensional turbulent convective plumes that flux buoyancy vertically and create a deep mixed patch. The lateral buoyancy gradient between the mixed patch and the ambient triggers a geostrophic current, which breaks down via baroclinic instability into discrete baroclinic eddies. These baroclinic eddies flux buoyancy laterally, arresting the growth of the mixed layer. The DNS is run over a range of linear background stratifications and buoyancy forcings. Increasing stratification flattens the slope of transient mixed layer deepening and suppresses convective deepening such that quasi-equilibrium mixed layer depth decreases with increasing stratification. Vertical diffusivity increases to a peak during the convective deepening phase of open-ocean convection, before dropping to a quasi-equilibrium value. The quasi-equilibrium vertical diffusivity in the DNS also decreases with increasing stratification, likely as a consequence of the suppression of convective plumes at higher stratification. Increasing the buoyancy forcing enhances both the vertical diffusivity and the quasiequilibrium mixed layer depth due to a strengthening of convective plumes in the system. Quasi-equilibrium and transient mixed layer growth in the DNS align with scaling predictions laid out by Visbeck et al. (1996) and Turner (1973), particularly at values of background stratification close to those in the Southern Ocean. The DNS results therefore justify the use of these scaling theories in large-scale ocean models.

The MOM6 simulations closely mimic the DNS during the growth of the diffusive mixed layer and the lateral spreading phase. However, during the deep convective growth of the mixed layer, the MOM6 simulations differ from the DNS in that they are entirely absent of turbulent convection. The vertical convection in the MOM6 simulations is instead laminar, with no three-dimensionality or anisotropy, in stark contrast to the vigorous, highly turbulent convection in the DNS results. The lack of turbulent convection in the MOM6 simulations has direct bearing on mixed layer depth in the model. While the quasi-equilibrium mixed layer depth in the MOM6 simulations matches the scaling predictions, the transient mixed layer growth during deep convection is very different. The mixed layer overshoots, becoming anomalously deep before stabilizing at a quasi-equilibrium depth. The overshoot in transient mixed layer depth is evident with both the ePBL and the KPP mixing schemes. We posit that the mixed layer depth bias in the MOM6 model is due to the lack of turbulent convection, which delays the onset of baroclinic instability, thereby allowing the mixed layer to grow too deep before eddies restratify the system.

The deep mixed layer bias in the MOM6 runs occurs during transient mixed layer deepening, which is particularly alarming given that most ocean models are forced with temporally varying (often seasonal) signals, and the system is not expected to reach quasiequilibrium. In addition, in the Southern Ocean, the anomalously deep mixed layer may penetrate the warm, salty Circumpolar Deep Water (CDW) that underlies the cool, fresh surface layer. The entrainment of the warm CDW toward the surface can inhibit sea ice formation and expose the surface water mass to atmospheric cooling. This destabilizing buoyancy signal in turn cools the warm CDW, resulting in the formation of deep convective cells in which warm CDW is entrained upward and cool surface waters are subducted through depth. These convective cells may extend through the entire water column and significantly alter the local ocean properties. Therefore, small variations in the transient mixed layer depth in the Southern Ocean can have a big impact on dense water formation and ocean ventilation. 
To address this bias, we suggest the incorporation of a stochastic parameterization of convection in large-scale ocean models. By seeding random turbulent variability in the momentum equations during open-ocean convection, the model will mimic the small-scale turbulence observed in the DNS and speed up the onset of baroclinic eddies in the system. The formulation of a new stochastic convective parameterization is beyond the scope of this paper and is reserved for future model development work.

The findings presented here highlight the importance of turbulence in modulating key flow features during open-ocean convection and propose a way to improve the representation of turbulent convection in future modeling studies. In addition, the DNS results validate existing scaling theories, providing further license to incorporate them in future model development efforts. However, there are a number of limitations in the present study that may be addressed in future work. The background stratification modeled here is simplistic - the ocean does not typically have a linear background stratification, and background stratification is significantly modified during preconditioning that is not modeled here. Openocean convection may therefore have a different response in the presence of more realistic background stratification conditions. The model has no background circulation and no bottom topography to steer the flow, and temporal and spatial variance in the surface buoyancy forcing is not represented. Variance in surface forcing is likely to occur in the ocean and may impact the quasi-equilibrium and transient mixed layer depth during open-ocean convection. That said, the flow solution in the DNS, even in the model's current simplistic configuration, aligns closely with observations of deep convection in the ocean. Therefore, investigations involving varying buoyancy profile, basal topography, or buoyancy forcing are reserved for future work.

Acknowledgments. Numerical simulations were conducted on the Australian National Computational Infrastructure (NCI), ANU, which is supported by the Commonwealth of Australia. B.G. was supported by the Australian Research Council (ARC) Future Fellowship (FT180100037). We thank two reviewers for their helpful feedback and advice.

\section{REFERENCES}

Adcroft, A., and Coauthors, 2019: The GFDL global ocean and sea ice model OM4.0: Model description and simulation features. J. Adv. Model. Earth Syst., 11, 3167-3211, https://doi.org/ 10.1029/2019MS001726.

Callies, J., and R. Ferrari, 2018: Baroclinic instability in the presence of convection. J. Phys. Oceanogr., 48, 45-60, https:// doi.org/10.1175/JPO-D-17-0028.1.
Cheon, W. G., Y.-G. Park, J. R. Toggweiler, and S.-K. Lee, 2014: The relationship of Weddell polynya and open-ocean deep convection to the Southern Hemisphere westerlies. J. Phys. Oceanogr., 44, 694-713, https://doi.org/10.1175/JPO-D-13-0112.1.

—, S. K. Lee, A. L. Gordon, Y. Liu, C. B. Cho, and J. J. Park, 2015: Replicating the 1970s' Weddell Polynya using a coupled ocean-sea ice model with reanalysis surface flux fields. Geophys. Res. Lett., 42, 5411-5418, https://doi.org/10.1002/ 2015 GL064364.

Clarke, R. A., and J.-C. Gascard, 1983: The formation of Labrador Sea Water. Part I: Large-scale processes. J. Phys. Oceanogr., 13, 1764-1778, https://doi.org/10.1175/1520-0485(1983)013<1764: TFOLSW $>2.0 . \mathrm{CO} ; 2$.

Coates, M. J., G. N. Ivey, and J. R. Taylor, 1995: Unsteady, turbulent convection into a rotating, linearly stratified fluid: Modeling deep ocean convection. J. Phys. Oceanogr., 25, 3032-3050, https://doi.org/10.1175/1520-0485(1995)025<3032: UTCIAR $>2.0 . \mathrm{CO} ; 2$.

Courtois, P., X. Hu, C. Pennelly, P. Spence, and P. G. Myers, 2017: Mixed layer depth calculation in deep convection regions in ocean numerical models. Ocean Modell., 120, 60-78, https:// doi.org/10.1016/j.ocemod.2017.10.007.

Cui, A., and R. L. Street, 2001: Large-eddy simulation of turbulent rotating convective flow development. J. Fluid Mech., 447, 5384, https://doi.org/10.1017/S0022112001006073.

Danabasoglu, G., S. G. Yeager, Y.-O. Kwon, J. J. Tribbia, A. S. Phillips, and J. W. Hurrell, 2012: Variability of the Atlantic meridional overturning circulation in CCSM4. J. Climate, 25, 5153-5172, https://doi.org/10.1175/JCLI-D-11-00463.1.

__ , and Coauthors, 2014: North Atlantic simulations in Coordinated Ocean-Ice Reference Experiments phase II (CORE-II). Part I: Mean states. Ocean Modell., 73, 76-107, https://doi.org/10.1016/j.ocemod.2013.10.005.

Farneti, R., and Coauthors, 2015: An assessment of Antarctic circumpolar current and Southern Ocean meridional overturning circulation during 1958-2007 in a suite of interannual CORE-II simulations. Ocean Modell., 93, 84-120, https://doi.org/10.1016/ j.ocemod.2015.07.009.

Ferrari, R., and F. Paparella, 2003: Compensation and alignment of thermohaline gradients in the ocean mixed layer. J. Phys. Oceanogr., 33, 2214-2223, https://doi.org/10.1175/ 1520-0485(2003)033<2214:CAAOTG $>2.0$. CO;2.

Fox-Kemper, B., R. Ferrari, and R. Hallberg, 2008: Parameterization of mixed layer eddies. Part I: Theory and diagnosis. J. Phys. Oceanogr., 38, 1145-1165, https://doi.org/10.1175/2007JPO3792.1.

Gelderloos, R., C. A. Katsman, and S. S. Drijfhout, 2011: Assessing the roles of three eddy types in restratifying the Labrador Sea after deep convection. J. Phys. Oceanogr., 41, 2102-2119, https://doi.org/10.1175/JPO-D-11-054.1.

Heidt, F. D., 1977: The growth of the mixed layer in a stratified fluid due to penetrative convection. Bound.-Layer Meteor., 12, 439461, https://doi.org/10.1007/BF00123192.

Holte, J., and L. Talley, 2009: A new algorithm for finding mixed layer depths with applications to argo data and subantarctic mode water formation. J. Atmos. Oceanic Technol., 26, 19201939, https://doi.org/10.1175/2009JTECHO543.1.

Ilicak, M., A. J. Adcroft, and S. Legg, 2014: A framework for parameterization of heterogeneous ocean convection. Ocean Modell., 82, 1-14, https://doi.org/10.1016/j.ocemod.2014.07.002.

Ivey, G. N., J. R. Taylor, and M. J. Coates, 1995: Convectively driven mixed layer growth in a rotating, stratified fluid. DeepSea Res. I, 42, 331-349, https://doi.org/10.1016/0967-0637(94) 00039-U. 
Jacobs, P., and G. N. Ivey, 1998: The influence of rotation on shelf convection. J. Fluid Mech., 369, 23-48, https://doi.org/10.1017/ S0022112098001827.

— vection in a homogeneous, rotating fluid. Dyn. Atmos. Oceans, 30, 149-171, https://doi.org/10.1016/S0377-0265(99)00024-X.

Jena, B., M. Ravichandran, and J. Turner, 2019: Recent reoccurrence of large open-ocean polynya on the Maud Rise seamount. Geophys. Res. Lett., 46, 4320-4329, https://doi.org/ 10.1029/2018GL081482.

Jones, H., and J. Marshall, 1997: Restratification after deep convection. J. Phys. Oceanogr., 27, 2276-2287, https://doi.org/ 10.1175/1520-0485(1997)027<2276:RADC>2.0.CO;2.

Julien, K., S. Legg, J. McWilliams, and J. Werne, 1996: Penetrative convection in rapidly rotating flows: Preliminary results from numerical simulation. Dyn. Atmos. Oceans, 24, 237-249, https://doi.org/10.1016/0377-0265(95)00449-1.

Katsman, C. A., M. A. Spall, and R. S. Pickart, 2004: Boundary current eddies and their role in the restratification of the Labrador Sea. J. Phys. Oceanogr., 34, 1967-1983, https:// doi.org/10.1175/1520-0485(2004)034<1967: BCEATR $>2.0 . \mathrm{CO} ; 2$.

Klinger, B. A., J. Marshall, and U. Send, 1996: Representation of convective plumes by vertical adjustment. J. Geophys. Res., 101, 18 175-18182, https://doi.org/10.1029/96JC00861.

Kostov, Y., K. C. Armour, and J. Marshall, 2014: Impact of the Atlantic meridional overturning circulation on ocean heat storage and transient climate change. Geophys. Res. Lett., 41, 2108-2116, https://doi.org/10.1002/2013GL058998.

Kuhlbrodt, T., and A. H. Monahan, 2003: Stochastic stability of openocean deep convection. J. Phys. Oceanogr., 33, 2764-2780, https:// doi.org/10.1175/1520-0485(2003)033<2764:SSOODC >2.0.CO;2.

Kurtakoti, P., M. Veneziani, A. Stössel, and W. Weijer, 2018: Preconditioning and formation of Maud Rise polynyas in a high-resolution Earth system model. J. Climate, 31, 9659-9678, https://doi.org/10.1175/JCLI-D-18-0392.1.

Large, W. G., J. C. McWilliams, and S. C. Doney, 1994: Oceanic vertical mixing: A review and a model with a nonlocal boundary layer parameterization. Rev. Geophys., 32, 363-403, https://doi.org/10.1029/94RG01872.

Legg, S., H. Jones, and M. Visbeck, 1996: A Heton perspective of baroclinic eddy transfer in localized open ocean convection. J. Phys. Oceanogr., 26, 2251-2266, https://doi.org/10.1175/ 1520-0485(1996)026<2251:AHPOBE >2.0.CO;2.

_ J. C. McWilliams, and J. Gao, 1998: Localization of deep ocean convection by a mesoscale eddy. J. Phys. Oceanogr. 28, 944-970, https://doi.org/10.1175/1520-0485(1998)028<0944: LODOCB $>2.0 . \mathrm{CO} ; 2$.

Levy, M. A., and H. J. S. Fernando, 2002: Turbulent thermal convection in a rotating stratified fluid. J. Fluid Mech., 467, 19-40, https://doi.org/10.1017/S0022112002001350.

Marshall, J., and F. Schott, 1999: Open-ocean convection: Observations, theory and models. Rev. Geophys., 37, 1-64, https://doi.org/10.1029/98RG02739.

Martinson, D. G., P. D. Killworth, and A. L. Gordon, 1981: A convective model for the Weddell polynya. J. Phys. Oceanogr., 11,
466-488, https://doi.org/10.1175/1520-0485(1981)011<0466: ACMFTW $>2.0 . \mathrm{CO} ; 2$.

Maxworthy, T., and S. Narimousa, 1994: Unsteady, turbulent convection into a homogeneous, rotating fluid, with oceanographic applications. J. Phys. Oceanogr., 24, 865-887, https://doi.org/ 10.1175/1520-0485(1994)024<0865:UTCIAH > 2.0.CO;2.

Noh, Y., W. G. Cheon, and S. Raasch, 2003: The role of preconditioning in the evolution of open-ocean deep convection. J. Phys. Oceanogr., 33, 1145-1166, https://doi.org/10.1175/ 1520-0485(2003)033<1145:TROPIT > 2.0.CO;2.

Reichl, B. G., and R. Hallberg, 2018: A simplified energetics based planetary boundary layer (ePBL) approach for ocean climate simulations. Ocean Modell., 132, 112-129, https://doi.org/ 10.1016/j.ocemod.2018.10.004.

Sallée, J.-B., R. J. Matear, S. R. Rintoul, and A. Lenton, 2012: Localized subduction of anthropogenic carbon dioxide in the Southern Hemisphere oceans. Nat. Geosci., 5, 579-584, https:// doi.org/10.1038/ngeo1523.

Sander, J., D. Wolf-Gladrow, and D. Olber, 1995: Numerical studies of open ocean deep convection. J. Geophys. Res., 100, 20 579-20 600, https://doi.org/10.1029/95JC02405.

Schmittner, A., 2005: Decline of the marine ecosystem caused by a reduction in the Atlantic overturning circulation. Nature, $\mathbf{4 3 4}$, 628-633, https://doi.org/10.1038/nature03476.

Schott, F., and K. D. Leaman, 1991: Observations with moored acoustic Doppler current profilers in the convection regime in the Golfe du Lion. J. Phys. Oceanogr., 21, 558-574, https://doi.org/ 10.1175/1520-0485(1991)021<0558:OWMADC >2.0.CO;2.

Send, U., and J. Marshall, 1995: Integral effects of deep convection. J. Phys. Oceanogr., 25, 855-872, https://doi.org/10.1175/15200485(1995)025<0855:IEODC>2.0.CO;2.

Sohail, T., B. Gayen, and A. M. Hogg, 2018: Convection enhances mixing in the Southern Ocean. Geophys. Res. Lett., 45, 41984207, https://doi.org/10.1029/2018GL077711.

— C. A. Vreugdenhil, B. Gayen, and A. M. Hogg, 2019: The impact of turbulence and convection on transport in the Southern Ocean. J. Geophys. Res. Oceans, 124, 4208-4221, https://doi.org/10.1029/2018JC014883.

Straneo, F., and M. Kawase, 1999: Comparisons of localized convection due to localized forcing and to preconditioning. J. Phys. Oceanogr., 29, 55-68, https://doi.org/10.1175/15200485(1999)029<0055:COLCDT > 2.0.CO;2.

Talley, L. D., 1999: Some aspects of ocean heat transport by the shallow, intermediate and deep overturning circulations. Mechanisms of Global Climate Change at Millennial Time Scales, Geophys. Monogr., Vol. 112, Amer. Geophys. Union, 1-22, https://doi.org/10.1029/GM112P0001.

Turner, J. S., 1973: Buoyancy Effects in Fluids. Cambridge University Press, 367 pp, https://doi.org/10.1017/CBO9780511608827.

Visbeck, M., J. Marshall, and H. Jones, 1996: Dynamics of isolated convective regions in the ocean. J. Phys. Oceanogr., 26, 1721-1734, https://doi.org/10.1175/1520-0485(1996)026<1721: DOICRI $>2.0 . \mathrm{CO} ; 2$.

Zwally, H. J., and P. Gloersen, 1977: Passive microwave images of the polar regions and research applications. Polar Rec., 18, 431-450, https://doi.org/10.1017/S0032247400000930. 